\title{
UDC 678
}

Author: MASKOVA Albina Rafitovna, PhD in Engineering, Associate Professor of Department «Applied and Natural Sciences»,Ufa State Petroleum Technological University; Mendeleev St., 195, Ufa, Bashkortostan Republic, Russia, 450080, asunasf@mail.ru;

Author: AMINOVA Guliya Karamovna, Doctor of Engineering, Professor of Department «Applied and Natural Sciences», Ufa State Petroleum Technological University; Mendeleev St., 195, Ufa, Bashkortostan Republic, Russia, 450080, aminovagk@inbox.ru;

Author: FAIZULLINA Svetlana Radikovna, Assistant of Department «Applied and Natural Sciences», Ufa State Petroleum Technological University; Mendeleev St., 195, Ufa, Bashkortostan Republic, Russia, 450080, sveta-mol86@mail.ru;

Author: FAIZULLINA Galiya Fatyhovna, Engineer of Department «Applied and Natural Sciences», Ufa State Petroleum Technological University; Mendeleev St., 195, Ufa, Bashkortostan Republic, Russia, 450080,galiya.aminova@gmail.com;

Author: MAZITOVA Aliya Karamovna, Doctor of Chemistry, Professor, Head of Department «Applied and Natural Sciences», Ufa State Petroleum Technological University; Mendeleev St., 195, Ufa, Bashkortostan Republic, Russia, 450080, elenaasf@yandex.ru

\section{PRODUCTION OF PVC-FILMS WITH SPECIFIC PROPERTIES}

\section{Extended Abstract:}

When developing polymeric materials with specified properties special compounds such as fungicides are used alongside traditional chemical additives (plasticizers). Usage of fungicides are determined by negative influence of mold fungi, bacteria and other microorganisms on polyvinyl chloride (PVC) material.

The present work focuses on the possibility to implement octylphenoxypropyl phthalates (OPOPP), proposed as plasticizers, and derivatives of 3-mercapto1,2,4-triazinon-5, proposed as fungicides, in PVC films. The first additive was produced by esterification of phthalic anhydride by oxypropylated phenols and 2-ethylhexanol, the second additive - by cyclocondensation of alpha-keto acids with thiosemicarbazide.

Having analyzed the results of experiments the authors found out that compositions obtained with the use of new additives - octylphenoxypropyl phthalates and derivatives of 3-mercapto-1,2,4-triazinone-5, acquire high performance 
properties that leads to increased service life. At the same time such important characteristics as strain at elongation, ultimate tensile stress, thermostability and funginertness are enhanced.

Key words: funginertness, dioctyl phthalate, tension at elongation, octylphenoxypropyl phthalate, ultimate tensile stress, PVC film, plasticizers of polyvinyl chloride, thermostability, fungicide.

MAChine-Readable information on CC-licenses (HTML-COdE) In METAdATA OF THE PAPER

$<$ a rel="license" href="http://creativecommons.org/licenses/by/4.0/" $><$ img alt="Creative Commons License" style="borderwidth:0" src="https://i.creativecommons.org/l/by/4.0/88x31.png" $/></ \mathrm{a}><$ br $/><$ span xmlns:dct="http://purl.org/ $\mathrm{dc} /$ terms/" href="http://purl.org/dc/dcmitype/Text" property="dct:title" rel="dct:type" $>$ Production of PVC-films with specific properties</span> by <a xmlns:cc="http://creativecommons.org/ns\#" href="Nanotehnologii v stroitel'stve = Nanotechnologies in Construction. 2018, Vol. 10, no. 4, pp. 102-115. DOI: dx.doi. org/10.15828/2075-8545-2018-10-4-102115" property="cc:attributionName" rel="cc:attributionURL">Maskova A.R., Aminova G.K., Faizullina S.R., Faizullina G. F., Mazitova A.K. </a $>$ is licensed under a $<$ a rel="license" href="http://creativecommons.org/licenses/by/4.0/" $>$ Creative Commons Attribution 4.0 International License $</ \mathrm{a}>$. $<$ br $/>$ Based on a work at $<$ a xmlns:dct="http://purl.org/dc/terms $/ "$ href=" http://nanobuild.ru/en_EN/nanobuild-4-2018/" rel="dct:source" $>$ http://nanobuild.ru/en_EN/nanobuild-4-2018/</ $\mathrm{a}>.<\mathrm{br} />$ Permissions beyond the scope of this license may be available at $<$ a xmlns:cc="http://creativecommons.org/ns\#" href="asunasf@mail.ru" rel="cc:morePermissions" $>$ asunasf@mail.ru</a> .

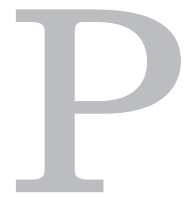

olymeric materials are used in different fields of industry. One can notice the increasing number of the works devoted to polymers with specified characteristics, as well as advanced requirements to be met by these materials.

At the present time polyvinyl chloride (PVC) is the most widely used polymer. Pure polyvinyl chloride is difficult processed, for this reason PVC is mixed with different additives. The main role of chemical additives is to facilitate polymeric mixtures processing and giving the necessary properties to finished products. A wide range of physical and mechanical properties of plastics are obtained by using additives, that makes it possible to produce a wide variety of products - from very soft, gel-like to elastic, rigid materials. Thus chemical additives expand the field of PVC applica- 
tion and allow it to be considered as a multicomponent system and the most easily compounded thermoplastic polymer [7-12].

More than 12 million tons of polymeric additives are produced every year in the world and plasticizers are the major part of them. Partial use of PVC in plasticized form are determined by characteristics of its chemicals structure - low elasticity, a significant temperature range of plastic deformation, and insufficient frost resistance [2, 5-7, 11, 12]. For this reason, intensification of manufacturing process and expansion of PVC application fields and providing wide variety of its properties, are connected to a large extent with the successful solution of the problem of creating effective plasticizers.

As known, phthalates are widely used as plasticizers in polymer industry. Dioctyl phthalate (DOP) is the main representative of phthalate plasticizers and it still remains the most widespread ester plasticizers of PVC. DOP possesses the optimal combination of properties, it is comparatively cheap, but DOP doesn't provide necessary service properties especially funginertness [11].

In this connection it is advantageous to produce the following additives: octylphenoxypropyl phthalates (OPOPP), which are proposed as plasticizers and derivatives of 3-marcapto-1,2,4-triazinone-5, which are proposed as fungicides. It is of great interest to study their properties and the possibility to develop PVC-materials that could meet the advanced operation requirements with the use of them.

We have produced new ester compounds - octylphenoxypropyl phthalates (OPOPP) with hydroxypropylation degree of phenol 1.0-2.1, that present slightly hygroscopic, oily liquids of yellowish color, highly soluble in organic solvents. OPOPP were obtained by the methods previously developed by us, by the esterification of phthalic anhydride by oxypropylated phenols and 2-ethylhexanol [13-27]. The physicochemical properties of proposed plasticizers are shown in table 1.

To determine the efficiency of suggested plasticizers the changing of Shor A hardness from the concentration of plasticizer were investigated (plasticizer: PVC-1:100) [2-6]. On the base of calculation of quantitative substitution factor (SF) it was determined that 1,0-OPOPP is very similar to DOP by plasticization efficiency (table 1).

As known, plasticized PVC materials can be exposed to various microorganisms including fungus under unfavorable conditions which adversely 
affected on the visual appearance of plastic compounds and reduce their service life. One of the way to protect PVC materials from negative influence of mold fungi is to introduce fungicides into compositions [11, 28].

Table 1

Physicochemical properties of octylphenoxypropyl phthalates

\begin{tabular}{|l|c|c|c|}
\hline \multicolumn{1}{|c|}{ Characteristics } & $\mathbf{1 , 0 - O P O P P}$ & $\mathbf{1 , 5 - O P O P P}$ & $\mathbf{2 , 1 - O P O P P}$ \\
\hline Yield, \% & 89 & 87 & 84 \\
\hline $\begin{array}{l}\text { Ester number., } \\
\text { mg KOH/g }\end{array}$ & 269 & 251 & 232 \\
\hline $\begin{array}{l}\text { Molecular mass, } \\
\text { found }\end{array}$ & 416 & 446 & 481 \\
\hline Flash point, ${ }^{\circ} \mathrm{C}$ & 199 & 198 & 198 \\
\hline Substitution factor & 1,019 & 1,043 & 1,052 \\
\hline
\end{tabular}

To realize this we obtained derivatives of 3 -mercapto-1,2,4-triazinones-5 (scheme 1):

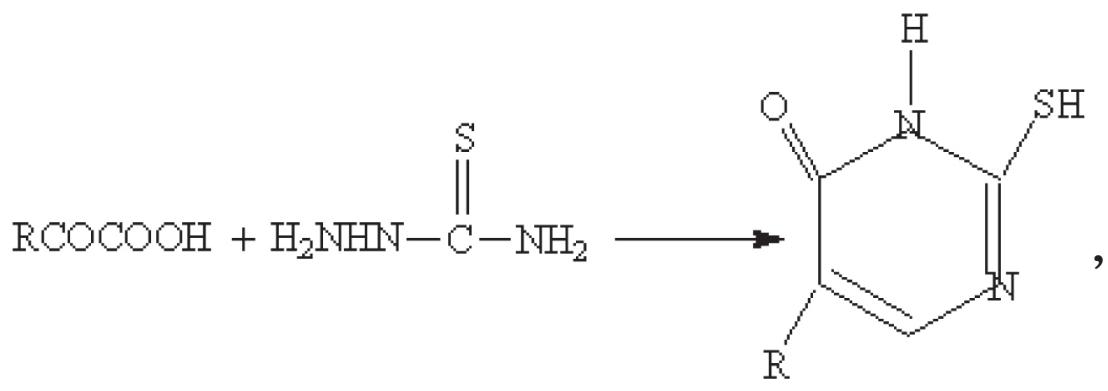

$(1-5)$

where $\mathrm{R}=\mathrm{CH}_{3}(1), \mathrm{i}-\mathrm{C}_{3} \mathrm{H}_{7}(2), \mathrm{i}-\mathrm{C}_{4} \mathrm{H}_{9}(3), \mathrm{t}-\mathrm{C}_{4} \mathrm{H}_{9}(4), \mathrm{C}_{6} \mathrm{H}_{5}(5)$.

Scheme 1

Compounds of the heterocyclic series were obtained by cyclocondensation of alpha-keto acids with thiosemicarbazide.

At the beginning, the reagents were loaded in the following order: water $100 \mathrm{ml}$ (solvent), sodium hydrogen carbonate 0,01 mole (cyclizing 
agent), alpha-keto acid 0,1 mole, thiosemicarbazide 0,1 mole. The reaction was performed at $100^{\circ} \mathrm{C}$ for 3 hours then cooled to $10^{\circ} \mathrm{C}$ and neutralized with concentrated hydrochloric acid to $\mathrm{pH}=6,0$. The obtained precipitate was filtrated, washed with water and dried on the air.

For obtaining 3-mercapto-1,2,4-triazinon-5 with the maximum yield, the filtrate was treated with a concentrated solution of sodium hydroxide and kept under synthesis conditions for 1 hour. The resulting precipitate was filtered off, washed with water and air-dried.

3-Mercapto-1,2,4-triazinones-5 are solids, from white to orange colour, soluble in many organic solvents.

Physicochemical properties and spectral characteristics of the synthesized compounds are presented in table 2.

Table 2

\section{Physicochemical properties and spectral characteristics of 3-mercapto-1,2,4-triazinones-5}

\begin{tabular}{|c|c|c|c|c|c|}
\hline \multirow{2}{*}{$\begin{array}{c}\text { Characteris- } \\
\text { tics }\end{array}$} & \multicolumn{5}{|c|}{ 3-Mercapto-1,2,4-triazinones-5 } \\
\hline & $\mathbf{1}$ & 2 & 3 & 4 & 5 \\
\hline Yield, \% & 80 & 80 & 74 & 79 & 80 \\
\hline $\begin{array}{l}\text { Melting } \\
\text { point, }{ }^{\circ} \mathrm{C}\end{array}$ & $217-219$ & $132-134$ & $160-162$ & $280-282$ & $255-257$ \\
\hline $\begin{array}{l}\text { IR-spec- } \\
\text { trum, sm }{ }^{-1}\end{array}$ & $\begin{array}{c}1670,1270 \\
1520\end{array}$ & $\begin{array}{c}1675,1270 \\
1540\end{array}$ & $\begin{array}{c}1690,1260 \\
1390\end{array}$ & $\begin{array}{c}1675,1260 \\
1520\end{array}$ & $\begin{array}{c}1670,1260 \\
1540\end{array}$ \\
\hline $\begin{array}{l}\text { UV-spec- } \\
\text { trum, nm }\end{array}$ & $190,275,310$ & $195,280,319$ & $190,270,315$ & $192,270,320$ & $220,290,330$ \\
\hline
\end{tabular}

Preliminary investigations have shown that derivatives of 3-mercapto-1,2,4-triazinon-5 possess high fungicidal activity [29, 30].

Next the produced compounds were tested in the PVC-films. Physical and mechanical properties of obtained PVC-films were analyzed according to standards: thermostability - according to GOST 14041-91, melt flow index - according to GOST 11645-73, funginertness - according to GOST 9.049-91, method 1 . 
The results of testing of plasticized PVC-films containing 3-mercapto-1,2,4-triazinones-5 as additive

\begin{tabular}{|c|c|c|c|c|c|c|}
\hline \multirow{2}{*}{ Characteristics } & \multirow{2}{*}{ Control $^{*}$} & \multicolumn{5}{|c|}{ 1,0-OPOPP + 3-mercapto-1,2,4-triazinones-5 } \\
\hline & & 1 & 2 & 3 & 4 & 5 \\
\hline $\begin{array}{l}\text { Strain at elongation } \\
100 \%, \mathrm{MPa}\end{array}$ & 11,1 & 11,9 & 11,8 & 12,0 & 12,2 & 12,4 \\
\hline $\begin{array}{l}\text { Ultimate tensile stress, } \\
\mathrm{MPa}\end{array}$ & 21,6 & 22,8 & 22,7 & 22,9 & 23,2 & 23,4 \\
\hline Breaking elongation, $\%$ & 340 & 305 & 305 & 309 & 308 & 310 \\
\hline $\begin{array}{l}\text { Extractability } \\
\text { by waters, \% }\end{array}$ & 0,012 & 0,212 & 0,243 & 0,146 & 0,087 & 0,014 \\
\hline Water absorption, \% & 0,195 & 0,312 & 0,315 & 0,294 & 0,207 & 0,201 \\
\hline Melt flow rate, $\mathrm{g} / 10 \mathrm{~min}$ & 44,5 & 40,2 & 40,4 & 39,3 & 38,9 & 38,7 \\
\hline $\begin{array}{l}\text { Thermostability } \\
\text { at } 175^{\circ} \mathrm{C} \text {, min }\end{array}$ & 165 & 170 & 169 & 172 & 174 & 175 \\
\hline Funginertness, grade & $\begin{array}{l}\text { Not re- } \\
\text { sistant }\end{array}$ & $\begin{array}{l}\text { Resis- } \\
\text { tant }\end{array}$ & $\begin{array}{l}\text { Resis- } \\
\text { tant }\end{array}$ & $\begin{array}{l}\text { Resis- } \\
\text { tant }\end{array}$ & $\begin{array}{l}\text { Resis- } \\
\text { tant }\end{array}$ & $\begin{array}{l}\text { Resis- } \\
\text { tant }\end{array}$ \\
\hline
\end{tabular}

* PVC-film, plasticized with DOP, without 3-mercapto-1,2,4-triazinones-5

As it can be seen from table 3, PVC-films produced by introducing developed octylphenoxypropyl phthalates and derivatives of 3-marcapto-1,2,4triazinone-5, acquire high performance properties that leads to increased service life of the films. In particular, one should note the improvement of the following indices: strain at elongation, ultimate tensile stress and thermostability. Evaluating funginertness of materials by the degree of fungi it has been found out that composition prepared on the base of PVC with octylphenoxypropyl phthalates and derivatives of 3-marcapto-1,2,4-triazinone-5 acquire high fungi resistance, while compositions prepared with the use of only plasticizer DOP were unstable with respect to microorganisms.

All this indicates the perspective of using octylphenoxypropyl phthalates as plasticizers and derivatives of 3-marcapto-1,2,4-triazinone-5 as fungicides. 
The performed research has been funded through the government grant «Synthesis and investigation of the modern polyvinyl chloride plasticizers» according to the Decree of the Government of the Republic of Bashkortostan of 07.02.2018 № 56 «On providing grants of the Republic of Bashkortostan to young scientists and groups of young researchers in 2018».

\section{References:}

1. Voskresenskyi V.A., Orlova E.M., Abramova E.I., Prohorova N.S. Plastifikaciya polimerov [Plasticization of polymer]. Uspehi himii [Russian chemical reviews]. 1971. Vol. 15. no. 1. pp. 142-159. (In Russian).

2. Shtarkman B.P. Plastifikatory PVH [PVC Plasticizers]. Moskow. Himija [Chemistry], 1975. 248 p. (In Russian).

3. Minsker K.S., Fedoseeva G.T. Destrukcija i stabilizacija polivinilhlorida [Degradation and stabilization of polyvinylchloride]. Moskow. Himija [Chemistry], 1979. 272 p. (In Russian).

4. Askadskiy A.A., Matveev Yu.I. Himicheskoe stroenie I fizicheskie svoistva polimerov [Chemical structure and physical properties of polymers]. Moskow. Himija [Chemistry], 1983. 248 p. (In Russian).

5. Uilki Ch., Sammers J., Daniels Ch. Polivinilhlorid [Polyvinylchloride]. Saint-Petersburg. Professija [Profession], 2007. 728 p. (In Russian).

6. Grossman F. Rukovodstvo po razrabotke kompozicii na ocnove PVH [Guidline for development of PVC compositions]. Moskow. Nauchnye ocnovy I tehnologii [Scientific foundation and technologies], 2009. 550 p. (In Russian).

7. Maslova I.P. Himicheskie dobavki k polimeram. Spravochnik [Chemical additives to polymers. Reference book]. Moskow. Himija [Chemistry], 1981. 264 p. (In Russian).

8. Mazitova A.K.,Aminova G.K., Nafikova R.F., Deberdeev R.Ja. Osnovnye polivinilhloridnye kompozicii stroitel'nogo naznachenija [Main polyvinylchloride compositions for building purposes]. Ufa, 2013. 130 p. (In Russian).

9. Mazitova A.K., Nafikova R.F., Aminova G.K. Plastifikatory polivinilhlorida [Plasticizers of polyvinylchloride] / Nauka i jepoha: monografija. Pod obshhej redakciej professora O.I. Kirikova [Science and epoch: monograph. Under the General editorship of Professor O. I. Kirikova]. Moskow; Voronezh, 2012. pp. 276-296. (In Russian).

10. Maskova A.R. Polivinilhloridnye kompozicii stroitel'nogo naznachenija, plastificirovannye ftalatami oksialkilirovannyh spirtov [Polyvinylchloride compositions for construction, plasticized by phthalates of alcohols oxyalkylated]: Dissertacija ... kand. tehn. nauk [The thesis for PhD in Engineering]. Ufa, 2012. 143 p. (In Russian).

11. Barshteyn R.S., Kirilovich V.I., Nosovskiy Y.E. Plastifikatory dlja polimerov [Plasticizers for polymers]. Moscow. Himija [Chemistry], 1982. 196 p. (In Russian). 
12. Tinius K. Plastifikatory [Plasticizers]. Moscow. Himija [Chemistry], 1964. 915 p. (In Russian).

13. Faizullina G.F., Gabitov A.I., Maskova A.R., Ahmetova I.I. Plastifikacija polivinilhlorida novymi plastifikatorami [Plasticization of polyvinylchloride with new plasticizers]. Neftegazovoe delo [Oil and gas business]. 2017. Vol. 15, no. 3. pp. 106-111. (In Russian).

14. Mazitova A.K., Aminova G.K., Maskova A.R., Yagafarova G.G., Mazitov R.M. New plasticizers for PVC-compositions in construction. Nanotehnologii v stroitel'stve $=$ Nanotechnologies in Construction. 2017, Vol. 9, no. 4, pp. 48-63. DOI: dx.doi.org/10.15828/20758545-2017-9-4-48-63.

15. Mazitova A.K., Aminova G.K., Maskova A.R., Zentsov V.N., Nedopekin D.V. and Rayzer Ju.S. 2017. Development of Oil-Benzen-Resistant PVC-Plastics. Journal of Engineering and Applied Sciences, 12: 7865-7869. DOI: 10.3923/jeasci.2017.7865.7869.

16. Mazitova A.K., Aminova G.K., Maskova A.R., Sabitov I.N., Nedoseko I.V. New polyvinylchloride plasticizers. Nanotehnologii v stroitel'stve $=$ Nanotechnologies in Construction. 2017, Vol. 9, no. 6, pp. 168-180. DOI: dx.doi.org/10.15828/2075-8545-2017-9-6-168-180.

17. Mazitova A.K., Aminova G.K., Gabitov A.I., Maskova A.R., Rahmatullina R.G. Novye plastifikatory PVH-kompozicij special'nogo naznachenija [New plasticizers of PVC compositions for special purposes]. Bashkir chemical journal [Bashkirsky Chemical Journal]. 2015. Vol. 22, no. 3. pp. 23-26. (In Russian).

18. Mazitova A.K., Aminova G.F. Gabitov A.I., Maskova, A.R., Khusnutdinov B.R., Fattakhova A.M. Razrabotka novyh plastifikatorov polivinilhlorida [Development of new plasticizers of polyvinyl chloride]. Jelektronnyj nauchnyj zhurnal «Neftegazovoe delo» [Electronic scientific journal «Oil and gas business»]. 2014. no. 12-1. pp. 120-127. (In Russian).

19. Mazitova A.K., Aminova G.K., Maskova A.R., Builova E.A., Nedopekin D.V. Difenoksijetilftalaty i butoksijetilfenoksijetilftalaty - novye plastifikatory polivinilhlorida [Diphenoxylate and butoxyethoxyethanol - new plasticizers of polyvinylchloride]. Jelektronnyj nauchnyj zhurnal «Neftegazovoe delo» [Electronic scientific journal «Oil and gas business»]. 2015. no. 5. pp. 376-397. (In Russian).

20. Mazitova A.K., Stepanova L.B., Aminova G.F., Maskova A.R. Razrabotka funkcional'nyh dobavok dlja polivinilhloridnyh kompozicij stroitel'nogo naznachenija [Development of functional additives for polyvinylchloride compositions for construction purposes]. Promyshlennoe proizvodstvo i ispol'zovaniejelastomerov [Industrial production and use of elastomers]. 2015. no. 2. pp. 27-31. (In Russian).

21. Maskova A.R., Stepanova L.B., Aminova G.F., Rol'nik L.Z., Abdrahmanova L.K. Ispytanie receptur PVH-kompozicij stroitel'nogo naznachenija na osnove novyh dobavok [Testing of formulations of PVC-compositions for construction purposes on the basis of new additives]. Promyshlennoe proizvodstvo i ispol'zovanie jelastomerov [Industrial production and use of elastomers]. 2015. no 3. pp. 11-15. (In Russian).

22. Aminova G.F., Gabitov A.I., Maskova A.R., Yagafarova G.G., Rolnik L.Z., Klyavlin M.S. New composite PVC-material for finishing purposes, plasticized by butoxyalkylphenoxyalkyl phthalates. Electronic scientific journal «Oil and gas business». 2013. no. 5. P. 353-362.

23. Mazitova A.K., Aminova G.K., Maskova A.R. Research of thermostability of phthalates 
of oxyalkylated alcohols. Nanotehnologii v stroitel'stve $=$ Nanotechnologies in Construction. 2018, Vol. 10, no. 2, pp. 157-170. DOI: dx.doi.org/10.15828/2075-8545-2018-102-157-170. (In Russian).

24. Maskova A.R., Mazitova A.K., Aminova G.K., Rolnik L.Z., Faizullina G.F. Investigation of the rheological properties of PVC compositions containing phthalate plasticizers. Nanotehnologii v stroitel'stve $=$ Nanotechnologies in Construction. 2018, Vol. 10, no. 3, pp. 127-137. DOI: dx.doi.org/10.15828/2075-8545-2018-10-3-127-137.

25. Maskova A.R., Builova E.A., Aznabaev S.T., Mazitova A.K. O nekotoryh svoistvah butoksijetilbnezoksijetilftalatov [About some properties of butoxyethylbenzoxyethyl phthalates]. Promyshlennoe proizvodstvo i ispol'zovanie jelastomerov [Industrial production and use elastomers]. 2015. no. 4. pp. 20-22. (In Russian).

26. Mazitova A.K., Nafikova R.F., Aminova G.K., Gabitov A. I., Maskova A.R., Khusnutdinov B.R. Polyvinylchloridnye kompozicii izolyacionnogo naznacheniya na ocnove ftalatov oksialkilirovannyh spirtov [Polyvinylchloride compositions for insulation purpose on the base of phthalates of oxyalkylated alcohol]. Promyshlennoe proizvodstvo $\mathrm{i}$ ispol'zovanie jelastomerov [Industrial production and use] elastomers]. 2014. no. 2. pp. 36-39. (In Russian).

27. Mazitova A.K., Aminova G.K., Maskova A.R., Builova E.A. Novye ftalatnye plastifikatory dlya polivinilhloridnogo linoleuma [New phthalate plasticizers for PVC-linoleum]. Jelektronnyj nauchnyj zhurnal «Neftegazovoe delo» [Electronic scientific journal «Oil and gas business»]. 2015. Vol. 13, no. 3. pp. 83-86. (In Russian).

28. Aleshin A.A., Panov Yu.T., Kudryavceva Z.A. Biorazrushaemaya polimernaya kompoziciiya [Biodegradable polymeric composition]. Sovremennye naukoemkie tehnologii [Modern high technologies]. 2007. no. 6. pp. 29-31. (In Russian).

29. Aminova G.K., Kudoyarova R.R, Bulgakov A.K., Mazitova A.K. O biologicheskoy aktivnosti 1,2,4-triazindionov-5,6 [About biological activity 1,2,4-triazindion-5,6]. Bashkirskii khimicheskii zhurnal [Bashkir Chemical Journal]. 2004. vol. 11. no. 4. pp.15-16. (In Russian).

30. Mazitova A.K., Sukhareva I.A ., Agzamov R.R., Buylova E.A., Nedopekin D.V. Himicheskie prevrashcheniya 3-merkaptoproizvodnyh nesimmetrichnyh triazinov [Chemical transformations of 3-mercaptoderivatives of asymmetric triazine]. Bashkirskii khimicheskii zhurnal [Bashkir Chemical Journal]. 2017. vol. 24, no. 2. pp.75-80. (In Russian).

\section{Dear COlleagues!}

THE REFERENCE TO THIS PAPER hAS THE FOLLOWING CITATION FORMAT:

Maskova A.R., Aminova G.K., Faizullina S.R., Faizullina G. F., Mazitova A.K. Production of PVC-films with specific properties. Nanotehnologii v stroitel'stve = Nanotechnologies in Construction. 2018, Vol. 10, no. 4, pp. 102-115. DOI: dx.doi. org/10.15828/2075-8545-2018-10-4-102-115. 
удК 678

Автор: МАСКОВА Альбина Рафитовна, к.т.н., доц. каф. «Прикладные и естественнонаучные дисциплины», ФГБОУ ВО «Уфимский государственный нефтяной технический университет»; ул. Менделеева, 195, г. Уфа, Республика Башкортостан, Россия, 450080, asunasf@mail.ru; Автор: АМИНОВА Гулия Карамовна, д.т.н., проф. каф. «Прикладные и естественнонаучные дисциплины», ФГБОУ ВО «Уфимский государственный нефтяной технический университет»; ул. Менделеева, 195, г. Уфа, Республика Башкортостан, Россия, 450080, aminovagk@inbox.ru; Автор: ФАЙЗУ ЛЛИНА Светлана Радиковна, ассистент каф. «Прикладные и естественнонаучные дисциплины», ФГБОУ ВО «Уфимский государственный нефтяной технический университет»; ул. Менделеева, 195, г. Уфа, Республика Башкортостан, Россия, 450080, sveta-mol86@mail.ru; Автор: ФАЙЗУ ЛЛИНА Галия Фатыховна, инженер каф. «Прикладные и естественнонаучные дисциплины», ФГБОУ ВО «Уфимский государственный нефтяной технический университет»; ул. Менделеева, 195, г. Уфа, Республика Башкортостан, Россия, 450080, galiya.aminova@gmail.com;

Автор: МАЗИТОВА Алия Карамовна, д.х.н., проф., зав. каф. «Прикладные и естественнонаучные дисциплины», ФГБОУ ВО «Уфимский государственный нефтяной технический университет»; ул. Менделеева, 195, г. Уфа, Республика Башкортостан, Россия, 450080, elenaasf@yandex.ru

\section{ПОЛУЧЕНИЕ ПВХ-ПЛЕНОК, ОБЛАДАЮЩИХ СПЕЦИФИЧЕСКИМИ СВОЙСТВАМИ}

АННОТАЦИЯ К СТАТЬЕ (АВТОРСКОЕ РЕЗЮМЕ, РЕФЕРАТ):

При разработке полимерных материалов, обладающих специфическими свойствами, наряду с применением традиционных химических добавок (пластификаторов) используются также и такие специальные вещества, как фунгициды. Применение последних обусловлено негативным воздействием на поливинилхлоридные (ПВХ) материалы плесневых грибов, бактерий и других микроорганизмов.

В настоящей работе исследована возможность практического применения октилфеноксипропилфталатов (ОФОПФ), предложенных в качестве пластификаторов, и производных 3-меркапто-1,2,4-триазинонов-5, предложенных в качестве фунгицидов, в ПВХ-пленках. Первая добавка получе- 
на этерификацией фталевого ангидрида оксипропилированными фенолами и 2-этилгексанолом, вторая - циклоконденсацией альфа-кетокислот с тиосемикарбазидом.

На основании проведенных экспериментов было установлено, что композиции, полученные с использованием новых добавок, а именно октилфеноксипропилфталатов и производных 3-меркапто-1,2,4-триазинонов-5, приобретают высокие эксплуатационные свойства, что приводит к увеличению срока их службы. При этом улучшаются основные показатели: напряжение при удлинении, разрушающее напряжение, термостабильность и грибостойкость.

Ключевые слова: грибостойкость, диоктилфталат, напряжение при удлинении, октилфеноксипропилфталат, разрушающее напряжение, ПВХ-пленка, пластификаторы поливинилхлорида, термостабильность, фунгициды.

DOI: dx.doi.org/10.15828/2075-8545-2018-10-4-102-115

МАШИНОЧИТАЕМАЯ ИНФОРМАЦИЯ О СС-ЛИЦЕНЗИИ В МЕТАДАННЫХ СТАТЬИ (HTML-КОД):

$<$ a rel="license" href="http://creativecommons.org/licenses/by/4.0/" $><$ img alt="Creative Commons License" style="borderwidth:0" src="https://i.creativecommons.org/l/by/4.0/88x31.png" / $></ \mathrm{a}><$ br $/><$ span xmlns:dct="http://purl.org/ dc/terms/" href="http://purl.org/dc/dcmitype/Text" property="dct:title" rel="dct:type">Production of PVC-films with specific properties $</$ span $>$ by $<$ a xmlns:cc="http://creativecommons.org/ns\#" href="Nanotehnologii v stroitel'stve = Nanotechnologies in Construction. 2018, Vol. 10, no. 4, pp. 102-115. DOI: dx.doi. org/10.15828/2075-8545-2018-10-4-102115" property="cc:attributionName" rel="cc:attributionURL">Maskova A.R., Aminova G.K., Faizullina S.R., Faizullina G. F., Mazitova A.K. $</ a>$ is licensed under a $<$ a rel="license" href="http://creativecommons.org/licenses/by/4.0/" $>$ Creative Commons Attribution 4.0 International License $</ \mathrm{a}>$. $<\mathrm{br} />$ Based on a work at $<$ a xmlns: $\mathrm{dct}=$ "http://purl.org/dc/terms $/ "$ href=" http://nanobuild.ru/en_EN/nanobuild-4-2018/" rel="dct:source" $>$ http://nanobuild.ru/en_EN/nanobuild-4-2018/</ a $>$. $<$ br $/>$ Permissions beyond the scope of this license may be available at $<$ a xmlns:cc="http://creativecommons.org/ns\#" href="asunasf@mail.ru"rel="cc:morePermissions" >asunasf@mail.ru</a>.

Работа выполнена при поддержке научного гранта «Синтез и исследование современных пластификаторов поливинилхлорида», в соответствии с Постановлением Правительства РБ от 07.02.2018 г. № 56 «О выделении в 2018 году грантов Республики Башкортостан молодым ученым и молодежным научным коллективам». 


\section{Библиографический список:}

1. Воскресенский В.А., Орлова Е.М., Абралов Е.И., Прохорова Н.С. Пластификация полимеров // Успехи химии. - 1971. - Т. 15, № 1. - С. 142-159.

2. Штаркман Б.П. Пластификация ПВХ. - М.: Химия, 1975. - 248 с.

3. Минскер К.С., Федосеева Г.Т. Деструкция и стабилизация поливинилхлорида. - М.: Химия, 1979. - 272 с.

4. Аскадский А.А., Матвеев Ю.И. Химическое строение и физические свойства полимеров. - М.: Химия, 1983. - 248 с.

5. Уилки Ч., Саллерс Дж., Даниелс Ч. Поливинилхлорид. - СПб.: Профессия, 2007. $728 \mathrm{c.}$

6. $\quad$ Гроссман Ф. Руководство по разработке композиций на основе ПВХ. - М.: Научные основы и технологии, 2009. - 550 с.

7. Маслова И.П. Химические добавки к полимерам. Справочник. - М.: Химия, 1981. $264 \mathrm{c}$.

8. Мазитова А.К., Алинова Г.К., Нафикова Р.Ф., Дебердеев Р.Я. Основные поливинилхлоридные композиции строительного назначения. - Уфа, 2013. - 130 с.

9. Мазитова А.К., Нафикова Р.Ф., Алинова Г.К. Пластификаторы поливинилхлорида // Наука и эпоха: монография. - под общей ред. проф. О.И. Кирикова. - Воронеж, 2011. - С. 276-296.

10. Маскова А.Р. Поливинилхлоридные композиции строительного назначения, пластифицированные фталатами оксиалкилированных спиртов: дис. ... канд. техн. наук. Уфа, 2012. - $143 \mathrm{c.}$

11. Барштейн Р.С., Кириллович В.И., Носовский Ю.Е. Пластификаторы для полимеров. М.: Химия, 1982. - 196 с.

12. Тиниус К. Пластификаторы. - М.: Химия, 1964. - 915 с.

13. Файзуллина Г.Ф., Габитов А.И., Маскова А.Р., Ахметова И.И. Пластификация поливинилхлорида новыми пластификаторами // Нефтегазовое дело. - 2017. - Т. 15, № 3. - С. 106-111.

14. Мазитова А.К., Алинова Г.К., Маскова А.Р., Ягафарова Г. Г., Мазитов Р.М. Новые пластификаторы для пвх-композиций строительного назначения // Нанотехнологии в строительстве. - 2017. - Том 9, № 4. - С. 48-63. - DOI: dx.doi.org/10.15828/20758545-2017-9-4-48-63.

15. Mazitova A.K., Aminova G.K., Maskova A.R., Zentsov V.N., Nedopekin D.V., Rayzer Ju.S. Development of Oil-Benzen-Resistant PVC-Plastics. Journal of Engineering and Applied Sciences, 12: 7865-7869. 2017. DOI: 10.3923/jeasci.2017.7865.7869.

16. Мазитова А.К., Алинова Г.К., Маскова А.Р., Сабитов И.Н., Недосеко И.В. Новые пластификаторы поливинилхлорида // Нанотехнологии в строительстве. - 2017. - Том 9, № 6. - C. 168-180. - DOI: dx.doi.org/10.15828/2075-8545-2017-9-6-168-180. 
17. Мазитова А.К., Алинова Г.К., Габитов А.И., Маскова А.Р., Рахматуллина Р.Г. Новые пластификаторы ПВХ-композиций специального назначения // Башкирский химический журнал. - 2015. - Т. 22, № 3. - С. 23-26.

18. Мазитова А.К., Алинова Г.Ф., Габитов А.И., Маскова А.Р., Хуснутдинов Б.Р., Фаттахова A.M. Разработка новых пластификаторов поливинилхлорида // Нефтегазовое дело. - 2014. - Т. 12, № 1. - С. 120-127.

19. Мазитова А.К., Алинова Г.К., Маскова А.Р., Буйлова Е.А., Недопекин Д.В. Дифеноксиэтилфталаты и бутоксиэтилфеноксиэтилфталаты - новые пластификаторы поливинилхлорида // Нефтегазовое дело. - 2015. - № 5. - С. 376-397.

20. Мазитова А.К., Степанова Л.Б., Алинова Г.Ф., Маскова А.Р. Разработка функциональных добавок для поливинилхлоридных композиций строительного назначения // Промышленное производство и использование эластомеров. - 2015. - № 2. - С. 27-31.

21. Маскова А.Р., Степанова Л.Б., Алинова Г.Ф., Рольник Л.З., Абдрахманова Л.К. Испытание рецептур ПВХ-композиций строительного назначения на основе новых добавок // Промышленное производство и использование эластомеров. - 2015. № 3. - C. 11-15.

22. Aminova G.F., Gabitov A.I., Maskova A.R., Yagafarova G.G., Rolnik L.Z., Klyavlin M.S. New composite PVC-material for finishing purposes, plasticized by butoxyalkylphenoxyalkylphthalates // Electronic scientific journal «Oil and gas business». - 2013. - № 5. P. 353-362.

23. Мазитова А.К., Алинова Г.К., Маскова А.Р. Исследование термостабильности фталатов оксиалкилированных спиртов // Нанотехнологии в строительстве. - 2018. Tом 10, № 2. - C. 157-170. - DOI: dx.doi.org/10.15828/2075-8545-2018-10-2-157-170.

24. Маскова А.Р., Мазитова А.К., Алинова Г.К., Рольник Л.З., Файзуллина Г.Ф. Исследование реологических свойств ПВХ-композиций, содержащих фталатные пластификаторы // Нанотехнологии в строительстве. - 2018. - Том 10, № 3. - С. 127-137. DOI: dx.doi.org/10.15828/2075-8545-2018-10-3-127-137.

25. Маскова А.Р., Буйлова Е.А., Азнабаев Ш.Т., Мазитова А.К. О некоторых свойствах бутоксиэтилбензоксиэтилфталатов // Промышленное производство и использование эластомеров. - 2015. - № 4. - С. 20-22.

26. Мазитова А.К., Нафикова Р.Ф., Алинова Г.Ф., Габитов А.И., Маскова А.Р., Хуснутдинов Б.Р. Поливинилхлоридные композиции изоляционного назначения на основе фталатов оксиалкилированных спиртов // Промышленное производство и использование эластомеров. - 2014. - № 2. - С. 36-39.

27. Мазитова А.К., Алинова Г.К., Маскова А.Р., Буйлова Е.А. Новые фталатные пластификаторы для поливинилхлоридного линолеума / Нефтегазовое дело. - 2015. - Т. 13, № 3. - С. 83-86.

28. Алешин А.А., Панов Ю.Т., Кудрявиева З.А. Биоразрушаемая полимерная композиции / Современные наукоемкие технологии. - 2007. - № 6. - С. 29-31. 
29. Алинова Г.К., Кудаярова Р.Р., Булдаков А.К., Мазитова А.К. О биологической активности 1,2,4-триазиндионов-5,6 // Башкирский химический журнал. - 2004. - Т. 11, № 4. - С. 15-16.

30. Мазитова А.К, Сухарева И.А., Агзалов Р.Р., Буйлова Е.А., Недопекин Д.В. Химические превращения 3-меркаптопроизводных несимметричных триазинов // Башкирский химический журнал. - 2017. - Т. 24, № 2. - С. 75-80.

УВАЖАЕМЫЕ КОЛЛЕГИ!

ПРИ ИСПОЛЬЗОВАНИИ МАТЕРИАЛА ДАННОЙ СТАТЬИ

ПРОСИМ ДЕЛАТЬ БИБЛИОГРАФИЧЕСКУЮ ССЫЛКУ НА НЕЁ:

Маскова А.Р., Алинова Г.К., Файзуллина С.Р., Файзуллина Г.Ф., Мазитова А.К. Получение ПВХ-пленок, обладающих специфическими свойствами // Нанотехнологии в строительстве. - 2018. - Том 10, № 4. - C. 102-115. - DOI: dx.doi.org/10.15828/2075-8545-2018-10-4-102-115.

\section{Dear colleagues!}

The REFERENCE TO THIS PAPER hAS THE FOLLOWING CITATION FORMAT:

Maskova A.R., Aminova G.K., Faizullina S.R., Faizullina G. F., Mazitova A.K. Production of PVC-films with specific properties. Nanotehnologii v stroitel'stve $=$ Nanotechnologies in Construction. 2018, Vol. 10, no. 4, pp. 102-115. DOI: dx.doi. org $/ 10.15828 / 2075-8545-2018-10-4-102-115$. 